\title{
Tuberkulose und Reisen ${ }^{*}$
}

\author{
Tuberculosis and Travelling
}

Autoren

Institute
G. Hoheisel ${ }^{1}$, M. Vogtmann ${ }^{2}$, C. Gessner ${ }^{1}$, J. Winkler ${ }^{1}$, A. C. Rodloff ${ }^{3}$, H. Wirtz ${ }^{4}$

Internistisch-Pneumologische Praxen, Leipzig

Klinik für Innere Medizin II (Gastroenterologie/Pneumologie), Park-Krankenhaus Leipzig-Südost GmbH

Institut für Medizinische Mikrobiologie und Infektionsepidemiologie, Universitätsklinikum Leipzig AöR

Department Innere Medizin, Abteilung Pneumologie, Universitätsklinikum Leipzig AöR eingereicht 7.4.2009

akzeptiert nach Revision 15.7. 2009

\section{Bibliografie}

DOI $10.1055 / \mathrm{s}-0029-1214995$

Pneumologie 2009; 63:

519-525 @ Georg Thieme

Verlag KG Stuttgart · New York

ISSN 0934-8387

\section{Korrespondenzadresse \\ Priv.-Doz. Dr. med. \\ Gerhard Hoheisel}

Innere Medizin, Pneumologie,

Allergologie

August-Bebel-Str. 69

04275 Leipzig

gerhard.hoheisel@t-online.de

\section{Zusammenfassung \\ $\nabla$}

Die Tuberkulose (TB) war im Jahre 2007 in Deutschland mit 5020 gemeldeten Fällen (Inzidenz: 6,1 Neu-Erkrankungen pro 100000 Einwohner) weiterhin rückläufig. 43,1\% dieser Personen kamen aus Ländern mit einer im Vergleich zu Deutschland höheren TB-Inzidenz. Doch nicht nur Migration, sondern auch Reisen von Menschen aus Niedrig- in Hoch-Inzidenz-Regionen der Welt sind mit einem erhöhten Risiko der Infektion mit M. tuberculosis (MTB) verbunden. Relativ selten kommt es zeitnah zu einer aktiven TB, häufiger jedoch zu einer latenten TB-Infektion (LTBI). Sowohl die akute TB als auch die LTBI, mit erst Jahre oder Jahrzehnte später möglicher Reaktivierung, können für den Einzelnen und das soziale Umfeld von erheblicher Bedeutung sein. Die frühzeitige Erkennung einer MTB-Infektion und deren mögliche Folgen sind für eine weiterhin erfolgreiche Bekämpfung tuberkulöser Erkrankungen entscheidend, insbesondere auch in Anbetracht des zunehmenden Reiseverkehrs.

\section{Einleitung}

$\nabla$

Die Inzidenz von Tuberkulose (TB) hat nach Angaben des Robert-Koch-Instituts (RKI) in den letzten zehn Jahren in Deutschland von 11163 Fällen im Jahre 1997 auf 5020 Fälle im Jahre 2007 (13,6 vs. 6,1 Neu-Erkrankungen pro 100000 Einwohner) abgenommen [1]. Obgleich der Anteil der im Ausland geborenen neu erkrankten Personen mit 43,1\% ( $n=2090)$ im Jahre 2007 vergleichsweise hoch ist, hat der Import von M. tuberculosis (MTB) als Folge der Immigration aus Hoch-InzidenzLändern vermutlich einen geringen Einfluss auf die Übertragung dieses Pathogens vor Ort $[1,2]$. So schien in einer Studie aus Hamburg eine erst kürzlich stattgefundene Übertragung im untersuchten Immigrantenkollektiv keine wesentliche Rolle gespielt zu haben, vielmehr scheint das Aus-

\section{Abstract \\ $\nabla$}

Tuberculosis (TB) in Germany in the year 2007 with 5020 reported cases (incidence: 6.1 newly diagnosed cases per 100000 inhabitants) was continuously in decline. $43.1 \%$ of these persons were from countries with a higher TB incidence as compared to Germany. However, not only migration but also personal journeys from low- to high-incidence countries carries an increased risk of infection with $M$. tuberculosis (MTB). An early active TB follows only rarely, more common, however, is a latent TB infection (LTBI). Not only the active form of TB but also LTBI, with a potential for reactivation years or decades later, can be of enormous relevance for the individual and the social environment. The early detection of an MTB infection and its possible sequelae are decisive for a continued successful battle against tuberculous diseases, especially in view of increasing travel activities.

maß einer Übertragung von einer Vielzahl unterschiedlicher Faktoren beeinflusst [3,4]. Doch nicht nur Migration, sondern auch Reisen von Menschen aus Ländern mit niedriger TB-Inzidenz, wie Deutschland, in Hoch-Inzidenz-Gebiete erscheinen bedeutsam, zumal viele dieser Regionen häufige und beliebte Reiseziele sind ( Tab. 1). So wird das Risiko einer MTB-Infektion bei LangzeitReisenden in Länder mit hoher TB-Inzidenz als erheblich eingeschätzt [5]. In einer eigenen Fallserie hatten vier von 75 Patienten eine TB-Infektion vermutlich auf Auslandsreisen erworben, 15 weitere wurden nachweislich von einem dieser Patienten infiziert; somit waren 25,3\% der Fälle direkt oder indirekt mit einer Auslandsreise in

\footnotetext{
* Herrn Professor Dr. med. Joachim Schauer, unserem klinischen Lehrer, zum 70. Geburtstag gewidmet.
} 
Tab. 1 Hoch-Inzidenz-Länder für Tuberkulose (TB) nach Schätzungen der WHO 2007 [7].

\begin{tabular}{|c|c|c|c|c|c|c|}
\hline \multirow[b]{3}{*}{ Land } & \multirow[b]{3}{*}{$\begin{array}{l}\text { Bevölkerung } \\
{[1000]}\end{array}$} & \multicolumn{4}{|l|}{ TB-Inzidenz ${ }^{\mathrm{a}}$} & \multirow{2}{*}{$\begin{array}{l}\text { HIV Prävalenz } \\
\text { bei TB-Fällen }\end{array}$} \\
\hline & & \multicolumn{2}{|c|}{ Alle TB-Formen } & \multicolumn{2}{|c|}{ mikroskopisch positiv } & \\
\hline & & Fälle [1000] & $\begin{array}{l}\text { pro } 100000 \\
\text { Einwohner/Jahr }\end{array}$ & Fälle [1000] & $\begin{array}{l}\text { pro } 100000 \\
\text { Einwohner/Jahr }\end{array}$ & $\%$ \\
\hline 1 Indien & 1169016 & 1962 & 168 & 873 & 75 & 5,3 \\
\hline 2 China & 1328630 & 1306 & 98 & 585 & 44 & 1,9 \\
\hline 3 Indonesien & 231627 & 528 & 228 & 236 & 102 & 3,0 \\
\hline 4 Nigeria & 148093 & 460 & 311 & 195 & 131 & 27 \\
\hline 5 Südafrika & 48577 & 461 & 948 & 174 & 358 & 73 \\
\hline 6 Bangladesh & 158665 & 353 & 223 & 159 & 100 & 0,3 \\
\hline 7 Äthiopien & 83099 & 314 & 378 & 135 & 163 & 19 \\
\hline 8 Pakistan & 163902 & 297 & 181 & 133 & 81 & 2,1 \\
\hline 9 Philippinen & 87960 & 255 & 290 & 115 & 130 & 0,3 \\
\hline 10 DR Kongo & 62636 & 245 & 392 & 109 & 174 & 5,9 \\
\hline 11 Russische Föderation & 142499 & 157 & 110 & 68 & 48 & 16 \\
\hline 12 Vietnam & 87375 & 150 & 171 & 66 & 76 & 8,1 \\
\hline 13 Kenia & 37538 & 132 & 353 & 53 & 142 & 48 \\
\hline 14 Brasilien & 191791 & 92 & 48 & 49 & 26 & 14 \\
\hline 15 UR Tansania & 40454 & 120 & 297 & 49 & 120 & 47 \\
\hline 16 Uganda & 30884 & 102 & 330 & 42 & 136 & 39 \\
\hline 17 Simbabwe & 13349 & 104 & 782 & 40 & 298 & 69 \\
\hline 18 Thailand & 63884 & 91 & 142 & 39 & 62 & 17 \\
\hline 19 Mozambique & 21397 & 92 & 431 & 37 & 174 & 47 \\
\hline 20 Myanmar & 48798 & 83 & 171 & 37 & 75 & 11 \\
\hline 21 Kambodscha & 14444 & 72 & 495 & 32 & 219 & 7,8 \\
\hline \multirow[t]{2}{*}{22 Afghanistan } & 27145 & 46 & 168 & 21 & 76 & 0 \\
\hline & 4201761 & 7423 & 177 & 3245 & 77 & 14 \\
\hline
\end{tabular}

a Patienten mit HIV eingeschlossen. ${ }^{b}$ Prävalenz von HIV bei TB-Fällen aller Altersgruppen. Nach Global tuberculosis control: surveillance, planning, financing : WHO report 2009 [7].

Verbindung zu bringen [6]. Der weltweite Anstieg therapieresistenter TB, die Komorbidität mit HIV wie z.B. in Südafrika $[7,8]$ und die zunehmende Mobilität des modernen Menschen zeigen die wachsende infektionsepidemiologische Bedeutsamkeit von Reiseaktivitäten und Infektionen mit MTB.

\section{Infektionsrisiko, Risikokollektive}

Das Spektrum reiseassoziierter Erkrankungen und die Häufigkeit der Fälle variiert je nach Land, Zusammensetzung der Bevölkerung und Art der berichtenden Institutionen ( $\bullet$ Tab. 2). Am häufigsten sind Malaria, Gastroenteritiden, Haut-, Harnwegs- und Atemwegsinfektionen. Die durch Aerosole übertragene TB ist mit $<4 \%$ aller Erkrankungen in den berichteten Serien allerdings relativ selten [7,9-11]. Vermutlich ist jedoch die Rate an Neu-Infektionen durch МТВ höher als die Zahl entdeckter TB, da diese im Gegensatz zu den meisten anderen reiseassoziierten Infektionen überwiegend unbemerkt verlaufen. Es besteht im Wesentlichen Übereinstimmung darüber, dass nur ein kleiner Teil derjenigen, die sich mit MTB infiziert haben, jemals an TB erkranken [12]. Risikokollektive sind in $\odot$ Tab. 3 aufgeführt. Von 622 Erkrankten, die nach einer Reise einen Arzt konsultierten, waren 45,3\% Touristen, 33,8\% Migranten nach dem Besuch ihrer Herkunftsländer, 14,1\% Angehörige ausländischer Institutionen („Expatriates“) und 6,7\% Geschäftsreisende [11]. Eine zunehmende Anzahl aus Entwicklungsländern stammender Migranten besuchen oftmals für längere Zeit ländliche Gebiete ihres Herkunftslandes und haben durch den engen Kontakt zur einheimi-
Tab. 2 Auf Reisen erworbene Erkrankungen [42].

\begin{tabular}{l} 
Atemwegsinfektion \\
Dengue-Fieber \\
Gastroenteritis \\
\hline Hautinfektion \\
Helminthose \\
Hepatitis A, B \\
Malaria \\
\hline Schistosomiasis \\
Tuberkulose \\
Typhus/Paratyphus \\
Venerische Infektionen/HIV
\end{tabular}

Tab. 3 Risiko-Kollektive.

Migranten auf Besuch im Herkunftsland
Geschäftsreisende
Touristen
Schüler/Studenten/Gastwissenschaftler („Auslandssemester“)
Mitarbeiter im Ausland für Firmen, Behörden, Hilfsorganisationen
(„Expatriates“)
Immunsupprimierte (einschließlich HIV, medikamentös bedingt)

schen Bevölkerung ein erhöhtes MTB-Infektionsrisiko [13]. Bei Touristen wird die Infektionsgefährdung auch durch das Bewusstsein des Gesundheitsrisikos beim Reiseveranstalter und besonders beim Reisenden selbst beeinflusst [14]. 
Tab. 4 Risikofaktoren.

Allgemeiner Immunstatus
TB-Immunstatus
BCG-Impfstatus
Reiseziel (Hoch-/Niedrig-Inzidenzgebiet)
Aufenthaltsdauer
Art der Tätigkeit (Gesundheitsberufe!)
Kontakt mit Tuberkulose-Kranken
Reisemittel (Kurz-/Langstreckenflug)
Art des Aufenthalts (z. B. Pilgerreise)
Art des Tourismus („Rucksack“, Hotel)

Tab.5 Häufigkeit nachgewiesener MTB-Infektionen von Reisenden.

\begin{tabular}{|lllrl}
$\mathbf{n}$ & $\begin{array}{l}\text { Detektions- } \\
\text { methode }\end{array}$ & $\begin{array}{l}\text { Infektionen } \\
(\mathbf{n})\end{array}$ & $\mathbf{( \% )}$ & Lit. \\
\hline 656 & THT & 12 & 1,8 & {$[5]$} \\
\hline 149 & IGRA & 15 & 10,0 & {$[29]$} \\
\hline
\end{tabular}

Das Risiko des Erwerbs einer MTB-Infektion ist abhängig vom Immunstatus des Reisenden, von der Wahrscheinlichkeit der Begegnung mit einer an infektiöser TB erkrankten Person, der Intensität und Dauer des Kontakts und von weiteren Faktoren in der Umgebung ( $\bullet$ Tab. 4). So hat ein „Rucksacktourist“, der ländliche Gebiete bereist und sehr engen Kontakt zur Lokalbevölkerung durch Transport in Bus, Bahn, Taxi und Übernachtung in einfacheren Unterkünften hat, vermutlich ein höheres Risiko eine MTB-Infektion zu akquirieren als der „Pauschalreisende“, der in Mehrsterne-Hotels wohnt und an organisierten Ausflügen mit nur begrenztem Kontakt zur einheimischen Bevölkerung teilnimmt. Es gibt nur wenige Untersuchungen zur Häufigkeit von Infektionen mit MTB während der Reisen ( $\bullet$ Tab. 5). In einer prospektiven Studie aus den Niederlanden mit nicht Bacillus-Calmette-Guérin-(BCG)-geimpften immunkompetenten Personen, die drei bis zwölf Monate in TB-Hoch-Inzidenz-Länder reisten, konnte mittels THT gezeigt werden, dass es in 1,8\% der Fälle während der Reise zu einer Infektion mit MTB gekommen war [5]. Das Risiko einer MTB-Infektion gilt für die meisten Reisenden als niedrig, erreicht aber bei Langzeit-Reisenden (> drei Monate) in Ländern mit hoher TB-Inzidenz das Risikoniveau der einheimischen Bevölkerung (0,5-2,5\% pro Jahr) [7,15].

Nach einer Infektion mit MTB kann es in wenigen Fällen zeitnah zu einer aktiven TB kommen. In den meisten Fällen verläuft eine Infektion während der Reisen vermutlich jedoch zunächst unbemerkt als latente tuberkulöse Infektion (LTBI), einem Zustand nach primärer MTB-Infektion mit Persistenz vitaler Bakterien im Organismus ohne Organbefund bzw. ohne Erkrankung [16]. Erst Jahre oder Jahrzehnte später kann es jedoch zur aktiven TB mit Gefährdung weiterer Personen im sozialen Umfeld kommen. Die Lebenszeitprävalenz einer aktiven TB bei Infizierten wird auf etwa $5-20 \%$ geschätzt $[17,18]$.

Kinder sind nach einer MTB-Infektion nicht nur besonders anfällig für eine aktive TB mit hoher Morbidität und Mortalität, vielmehr sind solche, die eine LTBI entwickeln auch Reservoir künftiger Übertragungen und Epidemien nach einer möglichen Reaktivierung im Erwachsenenalter [19-22]. Da nachgewiesene TB-Infektionen bei Kindern zumeist zeitnah erworben wurden, gelten sie als wichtiges Indiz einer MTB-Übertragung in der Algemeinbevölkerung und sind oftmals Anlass ausgedehnter Kontaktuntersuchungen zur Identifizierung infektiöser erwachsener Perso- nen im sozialen Umfeld [20]. Reisen von Kindern in ein Land mit hoher TB-Inzidenz oder Besuch von dort kommt eine besondere Bedeutung zu, da sich dadurch das Risiko des Erwerbs einer MTBInfektion erhöht. So hatten Kinder < 6 J., die in den letzten 12 Monaten ein Hochrisiko-Land besucht hatten, ein 3,9-fach erhöhtes Risiko für einen positiven THT im Vergleich zu nicht gereisten. Bei Kindern, deren Familien Besucher aus einem Hochrisiko-Land empfangen hatten, war das Risiko immerhin noch um das 2,4-fache erhöht [23]. Untersuchungen mittels THT und InterferonGamma-Release-Assays (IGRA) haben gezeigt, dass Personen mit nachgewiesener LTBI ein erhöhtes Risiko haben, eine aktive TB zu entwickeln $[20,24,25]$. Eine aktive Suche nach einer Reise mit nachgewiesenem oder vermutetem TB-Kontakt ist bei Kindern wegen der Verfügbarkeit einer prophylaktischen oder - bei Nachweis einer LTBI - präventiven Isoniazid-Therapie von besonderer Bedeutung [24]. Die derzeit gültigen Empfehlungen für Umgebungsuntersuchungen bei Kindern (s. Flussdiagramm $\bullet$ Abb. 1 a u. b) sollten genaueste Beachtung finden [26].

Das MTB-Infektionsrisiko von Personen, die in Gesundheitsberufen tätig sind, gilt als besonders hoch [15,27]. Dieser Beobachtung kommt besondere Bedeutung zu, da eine zunehmende Anzahl von Medizinstudentinnen und -studenten Abschnitte ihrer Ausbildung teils in Ländern mit hoher TB-Inzidenz absolvieren. So entwickelte ein Medizinstudent (24 J.) ohne sonstige Risikofaktoren, der einen mehrmonatigen Einsatz als Sanitäter im früheren Jugoslawien mitgemacht hatte, mehr als ein Jahr danach Luftnot und passagere Thoraxschmerzen, als deren Ursache eine TB Pleuritis diagnostiziert werden konnte [28]. In der bereits zitierten Studie aus den Niederlanden war die medizinische Versorgung von Patienten ein unabhängiger Risikofaktor für den Erwerb einer MTB-Infektion während eines längeren Auslandsaufenthaltes [5]. Ein hohes Infektionsrisiko hat jedoch nicht nur eine Tätigkeit in Gesundheitseinrichtungen, sondern vielmehr auch die Teilnahme an humanitären Hilfseinsätzen in Obdachlosenunterkünften, Armenvierteln, Gefängnissen sowie in Gebieten mit Bürgerkrieg, Unruhen und Flüchtlingslagern [7].

Auch riesige Menschenansammlungen wie bei Pilgerreisen bergen ein erhöhtes Risiko des Erwerbs einer MTB-Infektion. Unter den mehr als zwei Millionen Pilgern, die jährlich aus aller Welt nach Saudi Arabien reisen, finden sich TB-Erkrankungen häufig bei hospitalisierten Pilgern, darüber hinaus konnte mittels IGRA nachgewiesen werden, dass sich das Risiko des Erwerbs einer TB-Infektion durch die Reise erhöht [29].

Seit Jahrhunderten wird die TB auch als typische Erkrankung von Seeleuten betrachtet. In einer dänischen Studie wurden 64 Kultur-positive TB-Fälle von Seeleuten der Jahre 1992-2003 und die Übertragungswege einschließlich DNA-Subtypisierung analysiert. Das Risiko, an einer TB zu erkranken, war bei Seeleuten um das 1,5-fache im Vergleich zur Normalbevölkerung erhöht. Jedoch waren nur zwei der Fälle mit dem Seefahren assoziiert, fünf möglicherweise, der Rest unwahrscheinlich. Das erhöhte TB-Erkrankungsrisiko dieser Seeleute ist höchstwahrscheinlich durch in Dänemark erworbene Infektionen erklärbar. Diese Studie zeigt, dass es trotz multikultureller Mannschaften, teils aus Hoch-Inzidenz-Ländern, nur zu wenigen Übertragungen von MTB unter Seeleuten an Bord moderner Schiffe kommt [30]. 


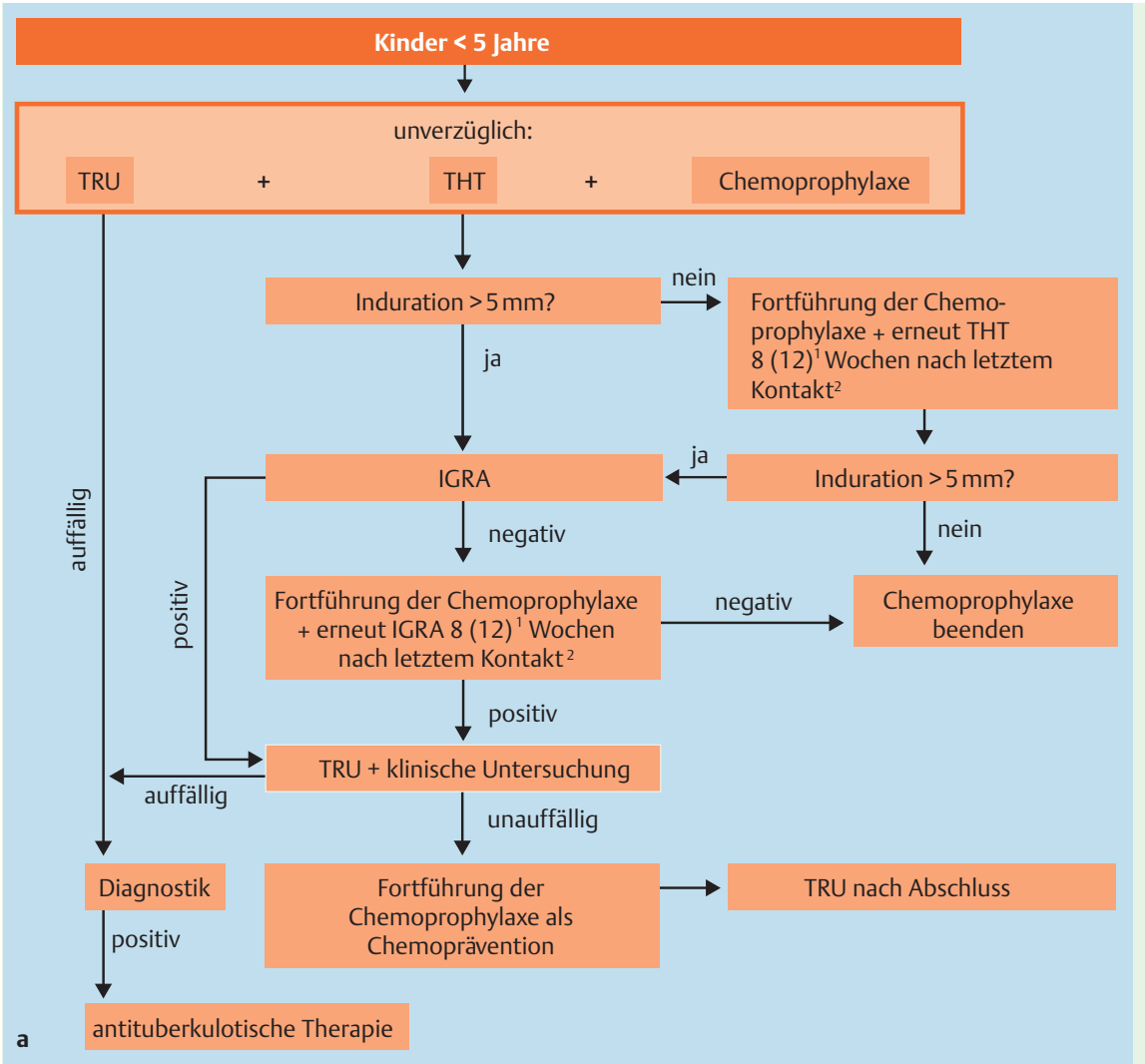

Abb. 1 a Flussdiagramm zur Umgebungsuntersuchung für enge Kontaktpersonen eines TB-Indexfalles: Kinder unter 5 Jahre.

${ }^{1}$ Die Empfehlung der Deutschen Gesellschaft für Pädiatrische Infektiologie (2003) sehen hiervon abweichend eine Chemoprophylaxe von zwölf Wochen und dementsprechend eine Nachtestung mit dem THT erst nach 12 Wochen vor.

${ }^{2}$ Wenn bei negativem IGRA nach initial positivem THT der letzte Kontakt zum Indexfall weniger als 8 (12) Wochen betragen hatte. (modifiziert nach [26])

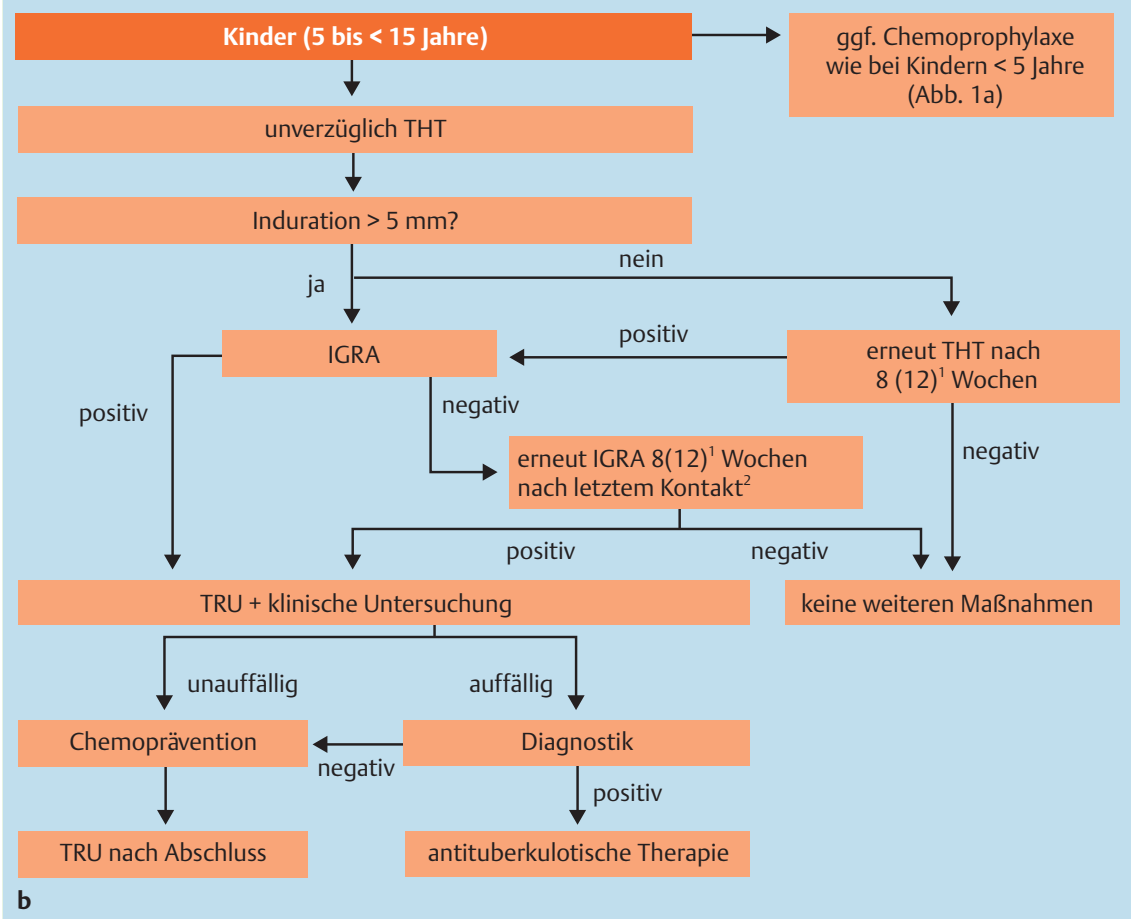

Abb. 1 b Flussdiagramm zur Umgebungsuntersuchung für enge Kontaktpersonen eines TB-Indexfalles: Kinder ( 5 bis > 15 Jahre).

${ }^{1}$ Die Empfehlung der Deutschen Gesellschaft für Pädiatrische Infektiologie (2003) sehen hiervon abweichend eine Nachtestung mit dem THT erst nach 12 Wochen vor.

${ }^{2}$ Wenn bei negativem IGRA nach initial positivem THT der letzte Kontakt zum Indexfall weniger als 8 (12) Wochen betragen hatte. Für Kontaktpersonen, die mit dem an einer im Sputumausstrich positiven Lungentuberkulose erkrankten Indexpatienten zusammenwohnen, ist die Indikation zur umgehenden TRU großzügig zu stellen. (modifiziert nach [26])

\section{Risiko bei Flugreisen} $\nabla$

Das Risiko der Übertragung respiratorischer Infektionen während der Flugreisen wurde mit dem Auftreten von SARS- und Influenza-Epidemien verstärkt untersucht. Mathematische Modelle, die die globale Ausbreitung solcher Infektionskrankheiten abbilden, betrachten ein Netzwerk internationaler Flughäfen als begünstigenden Faktor der Ausbreitung. So erscheint eine relativ kleine Anzahl von Flughäfen (200 bis 300 von insgesamt etwa
3000), die jedoch die größten Flughäfen zentraler Großstädte mit einbezieht, ausreichend, um die Dynamik einer globalen Ausbreitung zu beschreiben. Ein geringer Verkehr zwischen kleineren Flughäfen kann Störfaktoren vergrößern und andere Ausbreitungsmöglichkeiten, beispielsweise während des Transports von Passagieren am Boden, maskieren [31].

Auch die offene TB eines Passagiers stellt ein erhöhtes Infektionsrisiko für Mitreisende während langer Flüge dar [32 - 34]. Bei der aerogenen Übertragung von Krankheitserregern im Flugzeug ist 
Tab. 6 Screeningmethoden.

Anamnese
Tuberkulinhauttest (THT)
Interferon-Gamma-Release-Assays (IGRA)
Thorax-Röntgen-Untersuchung (TRU)
Mikrobiologische Diagnostik

zu beachten, dass die Belüftung an Bord so geplant ist, dass Luftbewegungen weitgehend auf die jeweiligen Kabinenabschnitte begrenzt werden. Studien haben gezeigt, dass MTB-Übertragungen von Passagier zu Passagier nur innerhalb desselben Kabinenabschnitts stattgefunden hatten. So wird angenommen, dass nur Passagiere, die in der Nähe des Indexfalles sitzen (je drei Sitzreihen nach vorne und hinten sowie drei Sitze zu jeder Seite abhängig von der Sitzanordnung des Flugzeugtyps), sowie das Begleitpersonal, welches in diesem Kabinenabschnitt Dienst hat, ein erhöhtes Infektionsrisiko hatten [35]. In einer Studie aus Neuseeland wurden Begleitpersonal und Reisende zweier Langstreckenflüge untersucht, bei denen jeweils ein Passagier mit offener TB an Bord war. 24 von 206 Passagieren hatten einen positiven THT, vier davon waren Konversionen. Alle diese Kontakte hatten Risikofaktoren für einen positiven THT, wie eine frühere BCG-Impfung oder Herkunft aus einem Land mit hoher TB-Inzidenz. Keine dieser Personen ist jedoch zu einem späteren Zeitpunkt an einer aktiven TB erkrankt [34]. In sieben Episoden mit Flugreisenden konnte mittels Kontaktuntersuchungen nur in zwei Fällen eine Infektion nachgewiesen werden, jedoch kam es bei keiner der infizierten Personen zu einer aktiven TB [36]. Multiresistente (MDR) TB-Fälle sind in den vergangenen Jahren zunehmend zu einem Gesundheitsproblem in vielen Ländern geworden, noch verstärkt durch das Auftreten extensiv resistenter (XDR) TB. Nach verschiedenen Fällen mit MDR- und XDR-TB bei Flugpassagieren wurde die WHO-Richtlinie über TB und Flugverkehr von 2006 angesichts neuer Erfahrungen und der zu erwartenden epidemiologischen Situation erweitert [37]. Eine Do-Not-Board(DNB)-Liste ermöglicht es dabei Gesundheitsbeamten in der ganzen Welt, Personen mit übertragbaren Erkrankungen, die bestimmte Kriterien erfüllen und ein ernsthaftes Risiko für die Öffentlichkeit darstellen, von Flügen von oder nach den USA auszuschließen. Eine erste Auswertung zeigte, dass Anträge zur Aufnahme in diese Liste für 42 Personen mit vermuteter oder bestätigter TB vorlagen, von denen dann 33 (79\%) in die Liste aufgenommen wurden [37]. Insgesamt zeigen die bisherigen Studien jedoch, dass eine Ausbreitung von Krankheitserregern einschließlich MTB in Flugzeugen nur selten vorkommt und nach den internationalen Richtlinien ein Infektionsrisiko in der Regel lediglich für Mitpassagiere desselben Kabinenabschnitts nach einer Flugdauer von mehr als acht Stunden besteht [7,26,38]. Die aktuellen Empfehlungen des RKI, des Deutschen Zentralkomitees zur Bekämpfung der Tuberkulose (DZK) und die aktuell geltenden gesetzlichen Bestimmungen sollten genaueste Beachtung finden.

\section{Prävention und Screening \\ $\nabla$}

Vor einer Reise sind Kenntnis des zu besuchenden Landes und des Infektionsrisiko bedeutsam. Eine BCG-Impfung für Reisende wird als wenig hilfreich zur Prävention einer TB gesehen und wird deshalb nicht empfohlen. In Ländern mit hoher TB-Inzidenz werden Kinder generell mit einer einzigen BCG-Dosis sobald wie möglich nach der Geburt geimpft. In den meisten Industriestaaten wurde diese Praxis jedoch eingestellt. Eine BCG-Impfung sollte allenfalls für Kinder aus Niedrig-Inzidenz-Länder bei Reisen in Hoch-Inzidenz-Regionen erwogen werden. Booster-Impfungen mit BCG werden nicht empfohlen [7]. Während einer Reise sollte der enge Kontakt zu bekannt an TB leidenden Personen vermieden werden [7].

Nach einer Reise sind dagegen Früherkennung und wirksame Behandlung einer LTBI oder aktiven TB bei symptomatischen Patienten oder bei Kontakt mit an TB Erkrankten entscheidend [39]. Risikofaktoren sollten gezielt abgefragt werden ( Tab.4). Als Werkzeuge zur Diagnose stehen verschiedene bewährte Methoden zur Verfügung ( Tab.6). Die vermehrte Reiseaktivität insbesondere junger Menschen sollte Anlass sein, bei der Anamneseerhebung, nach Reisen in Gebiete mit hoher TB-Inzidenz, nach der Art, Dauer und nach den benutzten Reisemitteln gezielt zu fragen und bei entsprechenden Symptomen eine TB differenzialdiagnostisch mit in Betracht zu ziehen [2]. Für Reisende aus Niedrig-Inzidenz-Ländern mit einer Tätigkeit, die mit einem erhöhten Risiko des Erwerbs einer TB assoziiert ist, sollte ein THT vor der Reise und erneut nach der Rückkehr angelegt werden [7]. Einen großen Fortschritt brachte die Etablierung der IGRA-Testverfahren zum Nachweis der in-vitro-Interferon-Gamma-Produktion sensibilisierter zirkulierender T-Lymphozyten 16-24 Stunden nach Kontakt mit MTB-spezifischen Antigenen [12,26, 29, 39, 40]. Die IGRA-Testverfahren haben eine höhere Spezifität in Bevölkerungsgruppen mit hoher Prävalenz von BCG-Impfungen im Vergleich zum THT [12]. Alle diese Methoden ergänzen sich, jedoch vermögen weder THT noch IGRA eine LTBI von einer aktiven TB sicher zu unterscheiden [12]. Die Thorax-Röntgen-Untersuchung (TRU) erlaubt bei entsprechenden Befunden die Verdachtsdiagnose einer aktiven TB und wird ggfs. ergänzt durch eine Computertomographie und weitere Untersuchungen. In einem Modell zur Kosteneffektivitäts-Analyse von TB-Screeningmethoden für Reisende in Hoch-Inzidenz-Länder wurde die Anwendung des THT nach der Reise in drei unterschiedlichen Strategien, zwei davon mit THT vor der Reise und TRU nach der Reise, miteinander verglichen. Berechnet wurden die möglicherweise entstehenden Kosten in einem Zeitraum von 20 Jahren, falls eine der Methoden Hinweise für eine LTBI ergeben und eine präventive Therapie angeboten werden würde. Es zeigte sich, dass ein einziger THT nach der Reise die effektivste Methode war, eine TB zu verhindern. Die Screeningmethoden waren am kosteneffektivsten für Reisende aus Niedrigrisiko- in Hochrisiko-Länder, je länger die Reise und je höher das Infektionsrisiko vor Ort waren. Die Studie evaluierte jedoch nicht die Anwendung des IGRA, der insbesondere für Reisende von Relevanz ist, die nach der Kindheit eine BCG-Impfung erhalten haben, wie dies in vielen europäischen Ländern der Fall ist. Der Entscheid zum Screening von Reisenden sollte die Dauer der Reise, die TB-Inzidenz und die Bereitschaft, eine LTBI zu behandeln, berücksichtigen [41]. Die derzeit gültigen Empfehlungen für Umgebungsuntersuchungen bei Kindern (s. o.) und bei Erwachsenen (s. Flussdiagramm $\bullet$ Abb. 2a u. b) sollten genaueste Beachtung finden [26]. Falls die THT-Reaktion und weitere Untersuchungen eine kürzliche Infektion nahe legen, sollte der Reisende eine präventive Therapie mit Isoniazid über mindestens neun oder besser zwölf Monate erhalten, da die Effektivität einer sechs-monatigen Therapie nur knapp $60 \%$ beträgt $[12,20]$. Die entsprechenden Leitlinien sollten genaue Anwendung finden $[16,26]$. 


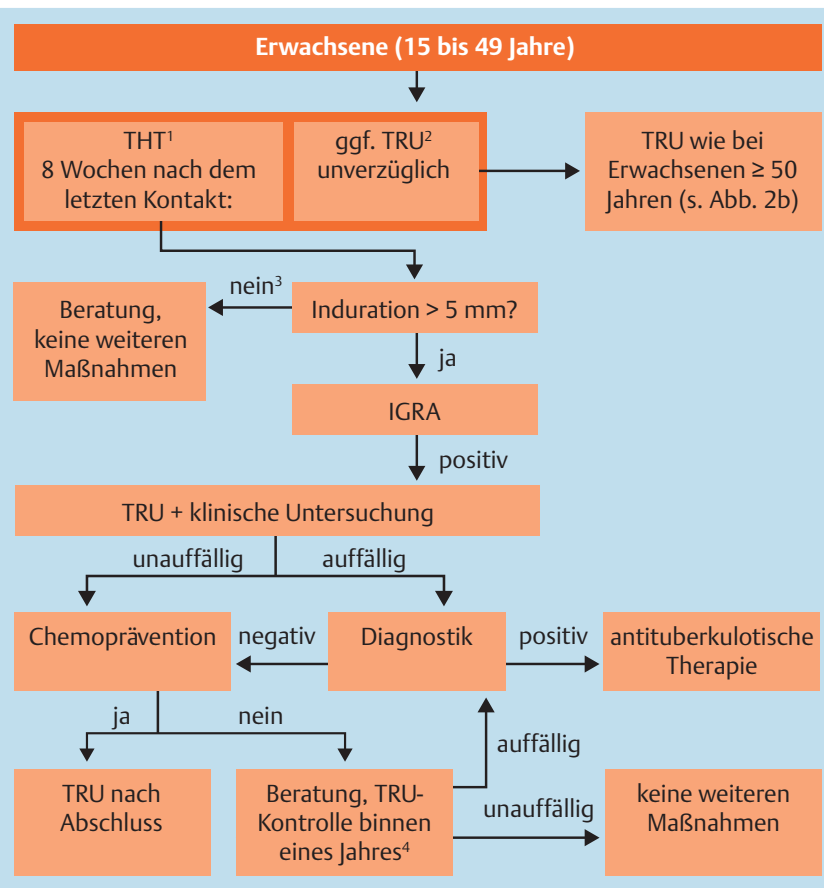

Abb. 2a Flussdiagramm zur Umgebungsuntersuchung für enge Kontaktpersonen eines TB-Indexfalles: Jugendliche und Erwachsene ( 15 Jahre bis 49 Jahre).

${ }^{1}$ Bei Akzeptanz bzw. sofern das Einhalten des Ablesezeitpunkts wahrscheinlich ist, sonst primär IGRA.

2 Einzelheiten s. [26].

${ }^{3}$ Ein negativer THT bei Immuninkompetenten schließt eine LTBI nicht aus, so dass hier auch primär oder im Anschluss ein IGRA zu erwägen ist.

${ }^{4}$ Einmalig, in der Regel nach 9 Monaten.

(modifiziert nach [26])

\section{Schlussfolgerung}

$\nabla$

Zunehmender internationaler Reiseverkehr und Migration haben zum Anstieg der TB-Raten in bestimmten Bevölkerungsgruppen in industrialisierten Ländern mit niedriger TB-Inzidenz geführt und drohen auch die Verbreitung multiresistenter MTB-Stämme zu fördern. Das Risiko einer aktiven TB nach einer MTB-Infektion während der Reisen in Hoch-Inzidenz-Länder erscheint gering, viel häufiger ist jedoch eine latente Infektion, die nach möglicher Reaktivierung oft Jahre oder Jahrzehnte später für den Einzelnen und das soziale Umfeld von erheblicher Bedeutung sein kann. Besonders gefährdet sind immunsupprimierte Personen, der längere Aufenthalt in Hoch-Inzidenz-Gebieten insbesondere bei Tätigkeit in medizinischen Berufen und bei engem Kontakt zur einheimischen Bevölkerung. Das Risiko einer Infektion mit MTB während eines Flugs erscheint gering. Eine BCG-Impfung wird im Allgemeinen nicht empfohlen. Dahingegen ist die aktive Suche zum Ausschluss oder Nachweis einer aktiven TB oder latenten Infektion nach einer Reise in Hoch-Inzidenz-Länder mit nachgewiesenem oder vermutetem TB-Kontakt bei Kindern und bei Erwachsenen von besonderer Bedeutung. Die entsprechenden Empfehlungen zur Umgebungsuntersuchung und $\mathrm{zu}$ einer prophylaktischen oder - bei Nachweis einer LTBI - präventiven IsoniazidTherapie sollten genaueste Beachtung finden.

\section{Interessenkonflikte}

Keine angegeben.

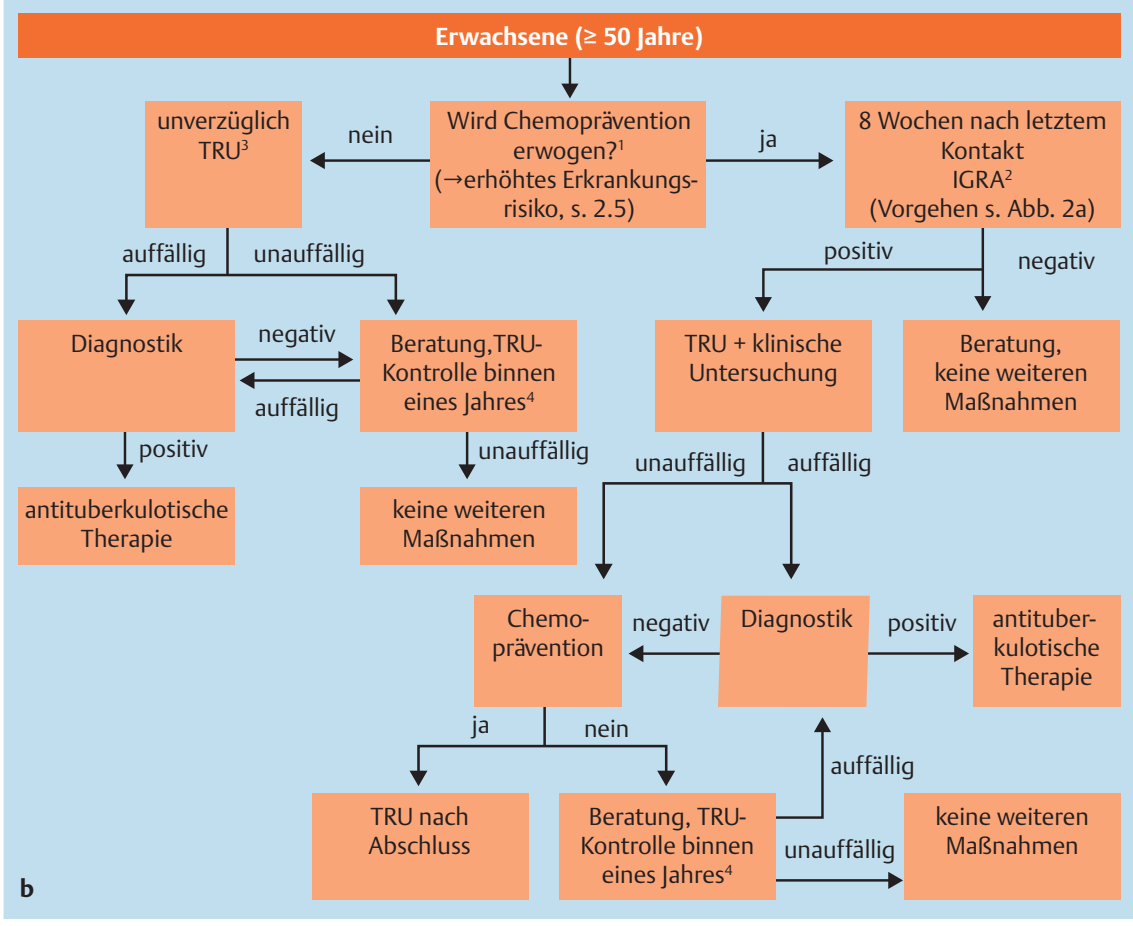

Abb. 2b Flussdiagramm zur Umgebungsuntersuchung für enge Kontaktpersonen eines TB-Indexfalles: Erwachsene ab 50 Jahre.

${ }^{1}$ Nach Nutzen-Risiko-Abwägung Indikationsstellung zur Chemoprävention nach entsprechender LTBIDiagnostik.

${ }^{2}$ Aufgrund der unzuverlässigeren Aussagekraft des THT im höheren Lebensalter und/oder bei Vorliegen von Risikofaktoren für ein falsch negatives THTErgebnis ist der initiale Einsatz eines IGRA sinnvoll, sofern verfügbar.

${ }^{3}$ Einzelheiten s. [26].

${ }^{4}$ Einmalig, in der Regel nach 9 Monaten. (modifiziert nach [26]) 


\section{Literatur}

1 Brodhun B, Altmann D, Haas W. Bericht zur Epidemiologie der Tuberkulose in Deutschland für 2007. Berlin: Robert Koch-Institut, 2009

2 Dahle UR, Eldholm V, Winje BA et al. Impact of immigration on the molecular epidemiology of Mycobacterium tuberculosis in a low-incidence country. Am J Respir Crit Care Med 2007; 176: 930 - 935

3 Diel R, Rüsch-Gerdes S, Niemann S. Molecular epidemiology of tuberculosis among immigrants in Hamburg, Germany. J Clin Microbiol 2004; 42: $2952-2960$

4 Lillebaek T, Andersen AB, Bauer J et al. Risk of Mycobacterium tuberculosis transmission in a low-incidence country due to immigration from high-incidence areas. J Clin Microbiol 2001; 39: 855-861

5 Cobelens FG, van Deutekom H, Draayer-Jansen IW et al. Risk of infection with Mycobacterium tuberculosis in travellers to areas of high tuberculosis endemicity. Lancet 2000; 356: 61 - 465

6 Hoheisel G, Vogtmann M, Winkler J, Gessner C, Hammerschmidt S, Seyfarth HJ, Reimann S, Rodloff AC, Gillissen A, Wirtz H. Spektrum von Tuberkuloseerkrankungen in der pneumologischen Praxis. Med Klin (München) 2008; 103: 769-777

7 WHO. Global Tuberculosis Control. Surveillance, planning, financing. WHO Report 2009. Geneva, Switzerland: WHO, 2009: 1 - 304. http:// www.who.int/tb/publications/global_report/2008/download _centre/ en/index.html

8 Gandhi NR, Moll A, Sturm AW et al. Extensively drug-resistant tuberculosis as a cause of death in patients co-infected with tuberculosis and HIV in a rural area of South Africa. Lancet 2006; 368: 1575-1580

9 Leroy H, Arvieux C, Biziragusenyuka J, Chapplain JM, Guiguen C, Michelet C, Tattevin P. A retrospective study of 230 consecutive patients hospitalized for presumed travel-related illness (2000-2006). Eur J Clin Microbiol Infect Dis 2008; 27: 1137-1140

10 O'Brien DP, Leder K, Matchett E, Brown GV, Torresi J. Illness in returned travelers and immigrants/refugees: the 6-year experience of two Australian infectious diseases units. J Travel Med 2006; 13: 145-152

11 Ansart S, Perez L, Vergely O, Danis M, Bricaire F, Caumes E. Illnesses in travellers returning from the tropics: a prospective study of 622 patients. J Travel Med 2005; 12: 312-318

12 Mack U, Migliori GB, Sester M et al. LTBI: latent tuberculous infection or lasting immune response to $\mathrm{M}$. tuberculosis? A TBNET consensus statement. Eur Respir J 2009; 33: 956-973

13 Fulford M, Keystone JS. Health Risks Associated with Visiting Friends and Relatives in Developing Countries. Curr Infect Dis Rep 2005; 7: $48-53$

14 Angell SY, Cetron MS. Health disparities among travellers visiting friends and relatives abroad. Ann Intern Med 2005; 142: 67 - 72

15 Johnston VJ, Grant AD. Tuberculosis in travellers. Travel Med Infect Dis 2003; $1: 205-212$

16 Schaberg T, Hauer B, Haas WH et al. Latente tuberkulöse Infektion: Empfehlungen zur präventiven Therapie bei Erwachsenen in Deutschland. Pneumologie 2004; 58: 255-270

17 Comstock GW, Livesay VT, Woolpert SF. The prognosis of a positive tuberculin reaction in childhood and adolescence. Am J Epidemiol 1974; 99: $131-138$

18 Vynnycky E, Fine PEM. Life time risks, incubation period, and serial interval of tuberculosis. Am J Epidemiol 2000; 152: 247-263

19 Newton SM, Brent AJ, Anderson S, Whittaker E, Kampmann B. Paediatric tuberculosis. Lancet Infect Dis 2008; 8: 498-510

20 Bakir M, Millington KA, Soysal A et al. Prognostic value of a T-cell-based, interferon-y-biomarker in children with tuberculosis contact. Ann Intern Med 2008; 149: 777 - 786
21 Marais BJ, Gie RP, Schaaf HS et al. Childhood pulmonary tuberculosis: old wisdom and new challenges. Am J Respir Crit Care Med 2008; 173: $1078-1090$

$22 \mathrm{Hsu} \mathrm{KH}$. Thirty years after isoniazid. Its impact on tuberculosis in children and adolescents. JAMA 1984; 251: 1283-1285

23 Lobato MN, Hopewell PC. Mycobacterium tuberculosis infection after travel to or contact with visitors from countries with a high prevalence of tuberculosis. Am J Respir Crit Care Med 1998; 158: 1871 - 1875

24 Kampmann B, Whittaker E, Williams A et al. Interferon-y-release assays do not identify more children with active tuberculosis than the tuberculin skin test. Eur Respir J 2009; 33: 1371 - 1379

25 Diel R, Loddenkemper R, Meywald-Walter K et al. Predictive value of a whole blood IFN-y assay for the development of active tuberculosis disease after recent infection with Mycobacterium tuberculosis. Am J Respir Crit Care Med 2008; 177: 1164-1170

26 Diel $R$, Forssbohm M, Loytved $G$ et al. Empfehlungen für die Umgebungsuntersuchungen bei Tuberkulose. Pneumologie 2007; 61: 440 455

27 Bowden K. Occupationally acquired tuberculosis: what's known. Journal of Occupational Medicine 1994; 36: 320-325

28 Vogtmann M, Beer J, Tannapfel A et al. Zur Differenzialdiagnostik und Therapie bei Husten, Fieber, Brustschmerz und einseitigem Pleuraerguss. Sächsisches Ärzteblatt 2004; 5: 191 - 194

29 Wilder-Smith A, Foo W, Earnest A, Paton NI. High risk of Mycobacterium tuberculosis infection during the Haij pilgrimage. Trop Med Int Health 2005; 10: $336-339$

30 Hansen HL, Henrik Andersen P, Lillebaek T. Routes of M. tuberculosis transmission among merchant seafarers. Scand J Infect Dis 2006; 38: $882 \mathrm{e}-887 \mathrm{e}$

31 Bobashev G, Morris RJ, Goedecke DM. Sampling for global epidemic models and the topology of an international airport network. PLoS ONE 2008; 3: e3154

32 Driver CR, Valway SE, Morgan WM et al. Transmission of Mycobacterium tuberculosis associated with air travel. JAMA 1994; 272: 1031 1035

33 Kenyon TA, Valway SE, Ihle WW et al. Transmission of multidrug-resistant Mycobacterium tuberculosis during a long airplane flight. N Engl J Med 1996; 334: 933 - 938

34 Whitlock G, Calder L, Perry H. A case of infectious tuberculosis on two long-haul aircraft flights: contact investigation. N Z Med J 2001; 114 $353-355$

35 Anonymous. Tuberculose et voyage. Med Mal Infect 2004; 34: 411 -414

36 Martinez L, Blanc L, Nunn P, Raviglione M. Tuberculosis and air travel: WHO guidance in the era of drug-resistant TB. Travel Med Infect Dis 2008; 6: 177-181

37 Centers for Disease Control and Prevention (CDC). Federal air travel restrictions for public health purposes - United States, June 2007 - May 2008. MMWR Morb Mortal Wkly Rep 2008; 57: 1009-1012

38 Leder $K$, Newman D. Respiratory infections during air travel. Intern Med J 2005; 35: 50 - 55

39 Lange C, Schaberg T, Diel R et al. Aktueller Stand der Tuberkulosediagnostik. Dtsch Med Wochenschr 2006; 131: $341-347$

40 Hauer B, Loddenkemper $R$, Detjen A et al. Interferon-gamma-Tests in der Tuberkulose-Diagnostik - Aktueller Stand. Pneumologie 2006; 60: $29-44$

41 Tan M, Menzies D, Schwartzman K. Tuberculosis screening of travellers to higher-incidence countries: a cost-effectiveness analysis. BMC Public Health 2008; 8: 201

42 WHO. International Travel and Health 2009. Geneva, Switzerland: WHO, 2009: 1 - 240. http://www.who.int/entity/ith/ITH_2009.pdf 